\title{
Zero Magnetization States in Electrodeposited Co0.45Fe0.55 Nanowire Arrays
}

\author{
Petru S. Fodor \\ Cleveland State University, p.fodor@csuohio.edu \\ Georgy M. Tsoi \\ Wayne State University \\ Lowell E. Wenger \\ Wayne State University, wenger@physics.wayne.edu
}

Follow this and additional works at: https://engagedscholarship.csuohio.edu/sciphysics_facpub

Part of the Physics Commons

How does access to this work benefit you? Let us know!

Publisher's Statement

(C) 2003 American Institute of Physics.

\section{Repository Citation}

Fodor, Petru S.; Tsoi, Georgy M.; and Wenger, Lowell E., "Zero Magnetization States in Electrodeposited Co0.45Fe0.55 Nanowire Arrays" (2003). Physics Faculty Publications. 210.

https://engagedscholarship.csuohio.edu/sciphysics_facpub/210

This Article is brought to you for free and open access by the Physics Department at EngagedScholarship@CSU. It has been accepted for inclusion in Physics Faculty Publications by an authorized administrator of

EngagedScholarship@CSU. For more information, please contact library.es@csuohio.edu. 


\section{Zero magnetization states in electrodeposited $\mathrm{Co}_{0.45} \mathrm{Fe}_{0.55}$ nanowire arrays}

P. S. Fodor, G. M. Tsoi, and L. E. Wenger

Citation: 93, 7035 (2003); doi: 10.1063/1.1557400

View online: http://dx.doi.org/10.1063/1.1557400

View Table of Contents: http://aip.scitation.org/toc/jap/93/10

Published by the American Institute of Physics

\section{Articles you may be interested in}

Long-range ordered nanoaperture array with uniform diameter and interpore spacing

87, 173116 (2005); 10.1063/1.2117608

A growth pathway for highly ordered quantum dot arrays

85, (2004); 10.1063/1.1834987

In situ fabrication of single-crystal Fe nanomagnet arrays 85, (2004); 10.1063/1.1803622

Periodic array of uniform $\mathrm{ZnO}$ nanorods by second-order self-assembly 84, (2004); 10.1063/1.1728298 


\title{
Zero magnetization states in electrodeposited $\mathrm{Co}_{0.45} \mathrm{Fe}_{0.55}$ nanowire arrays
}

\author{
P. S. Fodor, G. M. Tsoi, and L. E. Wenger ${ }^{\mathrm{a})}$ \\ Department of Physics and Astronomy, Wayne State University, Detroit, Michigan 48201
}

(Presented on 13 November 2002)

\begin{abstract}
$\mathrm{Co}_{0.45} \mathrm{Fe}_{0.55}$ alloy nanowires with 12 to $35 \mathrm{~nm}$ diameter and $12 \mu \mathrm{m}$ length were fabricated by electrodeposition in porous anodic alumina templates. The initial magnetization curves reveal that the zero magnetization state is not unique and is determined by the field history (ac demagnetization process) leading to the zero average moment state. For ac demagnetization processes with the field applied parallel to the nanowire axis, the subsequent magnetization curves suggest that an individual nanowire behaves as a single domain with neighboring nanowires being antiparallel to each other in the zero magnetization state. However, for a demagnetization process with the field applied perpendicular to the nanowires, a different zero magnetization state is created in which the individual nanowires consist of multidomains having opposite axial orientations. These results are consistent with the asymmetric (symmetric) behavior found in the minor hysteresis loops measured after perpendicular (parallel) ac demagnetization on these nanowire arrays. (C) 2003 American Institute of Physics. [DOI: 10.1063/1.1557400]
\end{abstract}

\section{INTRODUCTION}

The synthesis and characterization of magnetic nanostructures have been extensively investigated during the last decade due to an increasing demand for miniaturization and higher levels of performance capability in numerous applications. In addition to the more conventional techniques such as $\mathrm{x}$-ray and $e$-beam lithography which are able to produce state-of-the-art structures, ${ }^{1}$ a multitude of more novel nanofabrication methods have been developed including colloid chemical methods, ${ }^{2}$ atomic layer-by-layer deposition, ${ }^{3}$ selfassembly of polymers, ${ }^{4}$ and porous-membrane-based synthesis. ${ }^{5,6}$ From a more fundamental point of view, the interest focuses on the study of the new magnetic properties that emerge when the size of the nanostructures becomes comparable with the exchange lengths and domain wall thicknesses. For example, structures with large lateral confinement such as nanowires are expected to behave as single domain particles; ${ }^{7}$ however, recent magnetic force microscopy studies ${ }^{8}$ have shown that intricate domain structures can be created in a single nanowire.

In this paper we investigate the magnetic characteristics of electrodeposited $\mathrm{Co}_{0.45} \mathrm{Fe}_{0.55}$ alloy nanowire arrays in porous anodic alumina templates. Our previous studies on micrometer long Co-Fe alloy nanowires electrodeposited in alumina templates have shown that the magnetization reversal is a localized process. ${ }^{10}$ Once the magnetization switching takes place in a volume much smaller than the nanowire volume, there are no energy barriers to prevent the propagation of the reversal through the entire nanowire. As a consequence, the inhomogeneous magnetization states during the reversal process are short lived and the only stable magnetization states are single domains along the nanowire length, which is the easy axis resulting from the strong shape anisotropy. In this study evidence for stable multidomain structures

a)Electronic mail: wenger@physics.wayne.edu in the individual nanowires is presented on the basis of the magnetization curves measured from different zero magnetic moment states and minor hysteresis loops.

\section{EXPERIMENTAL DETAILS}

The electrodeposition of the magnetic transition metals and their alloys in porous membranes has proved to be a very simple and low-cost route for fabricating large arrays of magnetic nanowires. ${ }^{5,6,8-11}$ All of the samples reported in this study were prepared in porous anodic alumina templates since these templates can be produced with very uniform pore sizes and spacing. The $\mathrm{Co}_{0.45} \mathrm{Fe}_{0.55}$ nanowire arrays were fabricated in these hexagonally ordered templates by an ac electrodeposition technique as described more fully in Ref. 9. The pore spacing $D_{i}$ was fixed at $55 \mathrm{~nm}$ while the pore diameter $D_{p}$ was adjusted from 12 to $35 \mathrm{~nm}$ with the length of the nanowires being $12 \mu \mathrm{m}$.

All magnetic measurements were performed using a superconducting quantum interference device (SQUID) magnetometer. Both the initial magnetization curves and the minor hysteresis loops were measured with the field along the nanowires axis (normal to the Al substrate) starting from different initial demagnetized states. The demagnetized states were obtained through ac demagnetization processes beginning with fields larger than the coercive field; however, two different demagnetization (DM) processes were used. One of the demagnetization processes (parallel DM) had the demagnetizing field applied parallel to the length of the nanowires while the other (perpendicular DM) had the demagnetizing field applied perpendicular to the nanowire axis before magnetization measurements along the nanowire axis.

\section{RESULTS AND DISCUSSION}

Figure 1 shows the initial magnetization curves along the nanowire axis after the two demagnetization processes for $\mathrm{Co}_{0.45} \mathrm{Fe}_{0.55}$ alloy nanowire arrays with wire diameter $D_{p}$ 


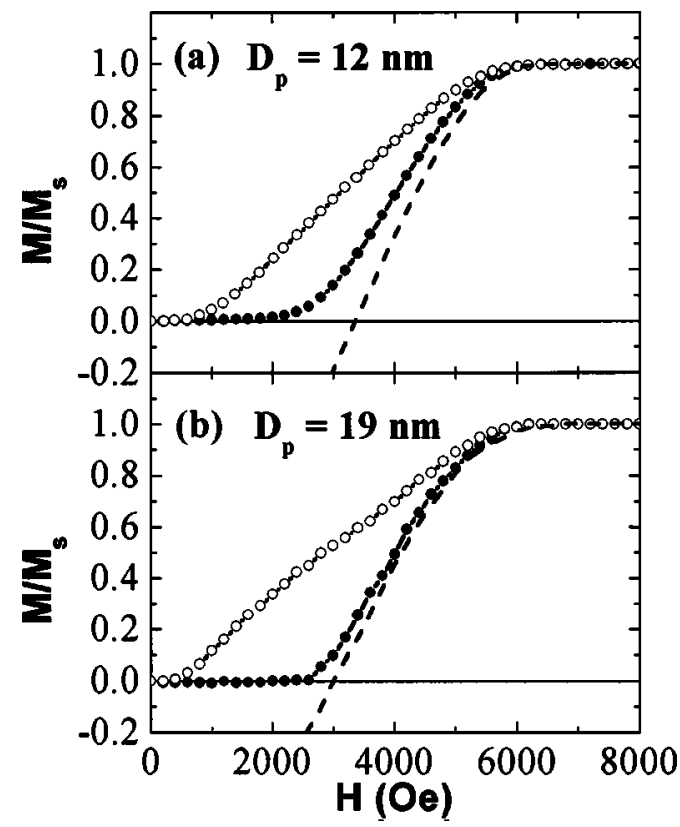

FIG. 1. Magnetization curves along the long axis for $\mathrm{Co}_{0.45} \mathrm{Fe}_{0.55}$ nanowire arrays with $D_{p}=$ (a) 12 and (b) $19 \mathrm{~nm}$. The samples were demagnetized with the magnetic field applied parallel $(\bullet)$ and perpendicular $(\bigcirc)$ to the nanowire length. The dashed line represents part of the total hysteresis loop.

$=12$ and $19 \mathrm{~nm}$. Since different initial magnetizations are observed depending on the field history, it is evident that the zero magnetic moment state is degenerate and depends on the field history as well. The magnetostatic interactions between the nanowires in such arrays are quite strong and a degeneracy of the zero moment state is expected because of the magnetic frustration present in this hexagonal structure. The situation is analogous to an antiferromagnetic Ising model on a triangular lattice where no configuration can be designed so that all the antiferromagnetic interactions are fully satisfied. As a result, there are a multitude of lowestenergy configurations for a zero moment state. However, since our magnetization measurements are performed on a very large number of nanowires representing a statistical average over a large number of possible configurations, no degeneracy should be expected. Therefore the large differences observed between the initial magnetization curves arise from differences in the magnetization structure of the individual nanowires that compose the arrays.

For samples demagnetized with the magnetic field parallel to the nanowire axis, the magnetization curves can be simply explained by considering each nanowire as a single domain with only two possible states: parallel (up) and antiparallel (down) to the nanowire axis. ${ }^{10}$ Clearly, the number of nanowires in the "up" state in a zero moment state is equal to the number of nanowires in the "down" state. As the external magnetic field is applied, no dramatic change in the magnetization state occurs until fields approach the value of the coercivity field. Then the internal magnetic fields are strong enough to activate the magnetization switching. In comparison, a large change in magnetization occurs even for small magnetic field values for samples demagnetized normal to the nanowires. This can be understood if multidomain magnetization states are initially present in each nanowire,

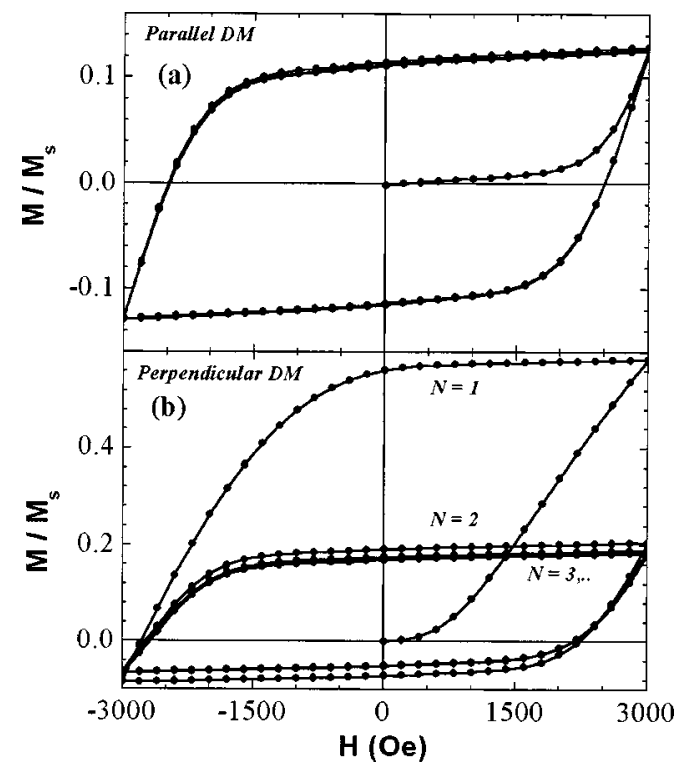

FIG. 2. Minor hysteresis loops for a $\mathrm{Co}_{0.45} \mathrm{Fe}_{0.55}$ nanowire array with $D_{p}$ $=12 \mathrm{~nm}$ for $H_{\max }=3000$ Oe. (a) Parallel and (b) perpendicular demagnetization.

which require no energy from the external field to create them. Thus applied fields much smaller than the coercivity field can change the multidomain magnetization states in a nanowire to a single-domain state and correspondingly a sizable magnetic response is measured. A recent magnetic force microscopy study on electrodeposited magnetic nanowires ${ }^{8}$ of similar diameter has shown that the magnetization structure for a single nanowire in the remanent state after the saturation field was applied normal to the nanowires consists of several axial domains separated by $180^{\circ}$ domain walls. This observation is a direct result of the large shape anisotropy and magnetization reversal localization. As the perpendicular magnetic field is removed, the magnetization in different parts of the nanowire aligns with the easy axis although there is no preferential orientation, and domain walls are formed at the boundaries between single domains. The net moment of an individual nanowire would not necessarily have to be zero. A similar process is thought to occur in the nanowire array structures during the demagnetization process when the field is applied perpendicular to the nanowire axis. The only substantive difference is that, since the nanowires in the array structures are magnetostatically coupled, these structures reach a local energy minimum in a zero moment state by cycling the demagnetization field.

Minor hysteresis loops were measured for these nanowire array structures in order to gain further insight into the dynamics of the states produced through the two different demagnetization processes. For parallel demagnetized samples, the minor hysteresis loops shown in Fig. 2 are symmetrical around the origin and reproducible for any recycling of the magnetic field. This result is not surprising for a system of quasi-identical magnetic units (single-domain nanowires) even at magnetic fields smaller than the saturation field. However, the behavior is dramatically different for samples demagnetized perpendicularly. Not only is the initial magnetic response larger, but the first $(N=1)$ minor hyster- 


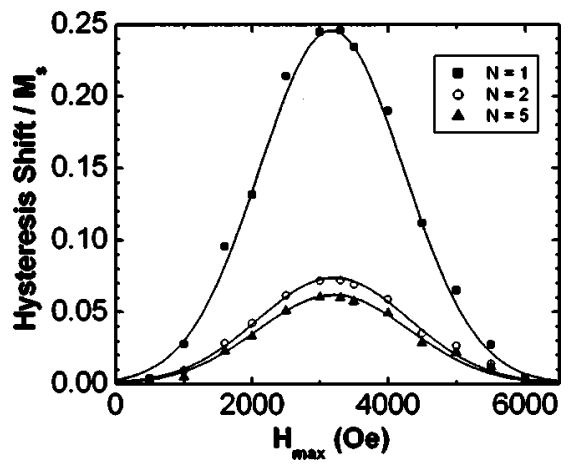

FIG. 3. Dependence of the center of the hysteresis loops for the $D_{p}$ $=12 \mathrm{~nm}$ array on the maximum applied magnetic field $H_{\max }$ and the number of cycles $N$. The lines are Gaussian fits to the data.

esis loop is not closed. It is only after a few magnetic field cycles that a closed hysteresis loop is achieved, with the center of the hysteresis loop showing a noticeable shift toward positive values of magnetization. Figure 3 more clearly displays this shift (midpoint of the hysteresis loop) as a function of the number of cycles and maximum applied field. We believe that this behavior arises from the strong irreversibility associated with the state of the system. While many nanowires having inhomogeneous (multidomain) states can be easily magnetized even in small fields, once the nanowires are in a single-domain state their magnetization can be reversed only if the applied field is larger than its coercivity field. Likewise, nanowires still remaining in inhomogeneous states after the initial maximum field $H_{\max }$ is applied will experience a magnetostatic interaction field from the surrounding fully magnetized (single-domain) nanowires that will tend to enhance the ability of these inhomogeneous nanowires to become fully magnetized in the opposite direction upon the reversal of the applied magnetic field. This process of fully magnetizing the nanowires into single domains continues through each cycle until all inhomogeneous states are essentially removed. Eventually, the coercivity of the individual nanowires and the magnetostatic interactions between the nanowires will dominate the characteristics of the minor hysteresis loops, just as in the parallel demagnetized case. In fact, the final closed minor hysteresis loops for the perpendicular demagnetized case are identical to those loops produced after the parallel demagnetization for the same $H_{\text {max }}$. The shift in the "center" of the initial hysteresis loops for $H_{\max }<H_{c}$ is related to the number of fully magnetized nanowires oriented "upward" during the first half of the cycle minus those oriented "downward" during the second half of each cycle. Not surprisingly, the maximum shift in these hysteresis loops occurs for loops having $H_{\max }$ approaching the measured coercivity, as approximately half of the nanowires with inhomogeneous states are fully magnetized during the first half of the first cycle for $H_{\max } \approx H_{c}$ $\left[M \approx(1 / 2) M_{s}\right]$ and the other half magnetized in the opposite direction for $H_{\max } \approx-H_{c}(M \approx 0)$, or a maximum shift of about $(1 / 4) M_{s}$. For $H_{\max }>H_{c}$, more than half of the nanowires are fully magnetized during the first half of the initial cycle, and the remainder are fully magnetized in the opposite direction during the subsequent second half of the cycle. In addition, a large fraction of the fully magnetized nanowires oriented upward have their moments reversed in the second half of the cycle since the "downward" applied field exceeds the coercivity field, thus lowering the center of the minor hysteresis loop toward zero.

In summary, evidence for inhomogeneous magnetization states in microscopic nanowires was found by performing macroscopic magnetization measurements. These states were obtained through a demagnetization process with the field applied perpendicular to the easy magnetization axis. The states are metastable, and small magnetic fields can induce irreversible changes toward the energetically favorable single-domain states.

\section{ACKNOWLEDGMENT}

This work is supported in part by the National Science Foundation through Grant No. DGE-9870720.

${ }^{1}$ S. Y. Chou, IEEE Trans. Magn. 85, 652 (1997).

${ }^{2}$ C. R. Martin, Science 266, 296 (1997).

${ }^{3}$ A. Gupta, Curr. Opin. Solid State Mater. Sci. 2, 23 (1997).

${ }^{4}$ S. I. Stupp, V. LeBonheur, K. Walker, L. S. Li, K. E. Huggins, M. Keser, and A. Amstutz, Science 276, 384 (1997).

${ }^{5}$ S. Dubois, C. Marchal, J. M. Beuken, L. Piraux, J. L. Duvail, A. Fert, J. M. George, and J. L. Maurice, Appl. Phys. Lett. 70, 396 (1997).

${ }^{6}$ H. Masuda, H. Asoh, M. Watanabe, K. Nishio, M. Nakao, and T. Tamamura, Adv. Mater. 13, 189 (2001).

${ }^{7}$ E. H. Frei, S. Shtrikman, and D. Treves, Phys. Rev. 106, 446 (1957).

${ }^{8}$ Y. Henry, K. Ounadjela, L. Piraux, S. Dubois, J. M. George, and J. L. Duvail, Eur. Phys. J. B 20, 35 (2001).

${ }^{9}$ P. S. Fodor, G. M. Tsoi, and L. E. Wenger, J. Appl. Phys. 91, 8879 (2002).

${ }^{10}$ P. S. Fodor, G. M. Tsoi, and L. E. Wenger (submitted for publication).

${ }^{11}$ L. Menon, S. Bandyopadhyay, Y. Liu, H. Zeng, and D. J. Sellmyer, J. Nanosci. Nanotechnol. 1, 149 (2001). 